\title{
miR-130a regulates macrophage polarization and is associated with non-small cell lung cancer
}

\author{
LIN LIN ${ }^{1}$, HAIBO LIN ${ }^{2}$, LIN WANG $^{1}$, BIN WANG $^{1}$, XUEZHI HAO ${ }^{1}$ and YUANKAI SHI ${ }^{1}$ \\ ${ }^{1}$ Department of Medical Oncology, Cancer Hospital Institute, Chinese Academy of Medical Science, Peking Union Medical \\ College, Beijing 100021; ${ }^{2}$ Department of Thoracic Surgery, Peking University, First Hospital, Beijing 100034, P.R. China
}

Received June 3, 2015; Accepted July 13, 2015

DOI: 10.3892/or.2015.4301

\begin{abstract}
Lung cancer is the most common cancer as well as the leading cause of cancer-related mortalities worldwide. Macrophages are the most abundant immune cells in primary and metastatic tumors, and contribute to tumor initiation, progression and metastasis. Macrophages have been shown to demonstrate a high level of plasticity, with the ability to undergo dynamic transition between M1 and M2 polarized phenotypes. In the present study, we investigated a pivotal role of miR-130a in macrophage polarization and whether it was associated with poor prognosis in non-small cell lung cancer (NSCLC), using RT-qPCR and western blot analyses. The in vitro experiments showed that miRNA-130a was expressed at a higher level in M1 compared to M2 macrophages. The enforced expression of miR-130a in macrophages resulted in a significantly increased production of proinflammatory cytokines, whereas deletion of miR-130a impaired the M2-associated gene expression and led to an M1-biased response. Mechanistically, the bioinformatics analysis revealed that proliferator-activated receptor $\gamma(\operatorname{PPAR} \gamma)$ is a potential target of miR-130a. Additionally, the luciferase assay confirmed that PPAR $\gamma$ translation was suppressed by miR-130a through the interaction with the 3'UTR of PPAR $\gamma$ mRNA. A subsequent analysis revaled that the induction of miR-130a suppressed PPAR $\gamma$ protein expression. In NSCLC patients, the results showed that miR-130a downregulation exhibited clinical relevance as it was correlated with poor prognosis and increased tumor stage and metastasis. In addition, miR-130a was inversely correlated with the macrophage marker, CD163, and target gene, PPAR $\gamma$. Taken together, the results established miR-130a as a molecular switch during macrophage development and as a potential target for the treatment of NSCLC.
\end{abstract}

Correspondence to: Dr Yuankai Shi, Department of Medical Oncology, Cancer Hospital Institute, Chinese Academy of Medical Science, Peking Union Medical College, 17 South Panjiayuan Lane, Beijing 100021, P.R. China

E-mail: pro_ykshi@126.com

Key words: non-small cell lung cancer, miR-130a, macrophages, polarization

\section{Introduction}

Lung cancer is the most common cancer as well as the leading cause of cancer-related mortalities globally (1). Histologically, non-small cell lung cancer (NSCLC) accounts for $\sim 80 \%$ of lung cancer cases. In spite of the emergence of new cytotoxic drugs and targeted biological agents, NSCLC remains one of the most clinically challenging cancer types (2). Thus, providing better treatment strategies is crucial.

Tumors are composed of an array of cell types, including cancer and non-cancer cells. The most prominent component of these non-cancer cells are macrophages, also known as tumor-associated macrophages (TAMs) (3). There are two well-established polarized phenotypes, classically activated macrophages (M1) and alternatively activated macrophages (M2), both of which have been observed in many tumors (4-6). M1-type macrophages are known to be induced by granulocytemacrophage colony-stimulating factor (GM-CSF), interferon $\gamma$ (IFN- $\gamma$ ), and/or lipopolysaccharide (LPS) and have an IL-12 ${ }^{\text {high }}$, IL-23 $3^{\text {high }}$ and IL-10 ${ }^{\text {low }}$ phenotype (7). At the opposite extreme, M2-type macrophages are various forms of macrophages other than the classic M1 including cells exposed to IL-4, IL-13, immune complexes, IL-10, and glucocorticoids (8). Generally, M1-type macrophages are regarded as the effector cells that defend the body against pathogens and tumor cells, while M2-type macrophages suppress inflammatory responses and adaptive immunity and stimulate angiogenesis and tumor growth $(9,10)$. The prognosis of cancer patients is dependent on the ratio of M1 and M2 macrophages $(11,12)$. TAMs play a pivotal role in the progression of NSCLC. The cytotoxic M1 phenotype explains the extended survival of patients with NSCLC, suggesting that positive immunoresponses play a crucial role in the prevention of NSCLC progression (13-16). Therefore, the molecular mechanisms underlying TAM polarization to different phenotypes are the focus of intense investigation.

microRNAs (miRNAs) have been shown to be important mediators of the macrophage activation process. miRNAs are a small class of nucleic acids ( $20-24 \mathrm{nt})$ that function in the transcriptional and post-transcriptional regulation of gene expression (17). miRNAs play a vital role in the regulation of most biological and physiological processes, including development, cell proliferation, cell cycle, apoptosis, migration, and differentiation, including those connected to cancer and 
Table I. Primer sequences used for RT-qPCR.

\begin{tabular}{lll}
\hline Gene & \multicolumn{1}{c}{ Sense primer } & \multicolumn{1}{c}{ Antisense primer } \\
\hline$I L-1 \beta$ & 5'-TCCAGGGACAGGATATGG AG-3' & 5'-TCTTTCAAC ACGCAGGACAG-3' \\
$T N F-\alpha$ & 5'-CTGTAGCCCATGTTGTAGCAAAC-3' & 5'-GCTGGTTATCTCTCAGCTCCAC-3' \\
$I L-10$ & 5'-GCCAAGCTGAAATTGAATGAGGA-3' & 5'-TTCTGTGCCGGCAGCTTTAAC-3' \\
$C C L 17$ & 5'-TTTAAGGGTTACCTGGGTTGC-3' & 5'-TTGATGTCTGGGTCTTGGTTC-3' \\
$C C L 22$ & 5'-GGATGCCATCGTTTTTGTAACTG-3' & 5'-AACTGCATTCTTCACTCTCTTGTTGT-3' \\
$C D 163$ & 5'-TGCCGTGATTACGTCCGTTA-3' & 5'-TCTCCTTATCCCTGAAGGTTAGCA-3' \\
$P P A R \gamma$ & 5'-GCTGCAGTGAATTGCACAGATAT-3' & 5'-CGGGATGAGCGACCTGTT-3' \\
$G A P D H$ & 5'CATGGTGCCTTCGCTGAT-3' & 5'-CAATGGCCATGAGGGAGTTA-3' \\
& 5'-GCACCGTCAAGGCTGAGAAC-3' & 5'-TGGTGAAGACGCCAGTGGA-3'
\end{tabular}

immunity (18-20). The role of miRNAs in the regulation of macrophage polarization has been largely undefined.

In the present study, we examined the role miR-130a played in the regulation of macrophage activation. The results showed that M1 macrophage has an elevated expression of miR-130a compared to M2. miR-130a suppressed the polarization of macrophages to the M2 phenotype and enhanced M1 polarization. miR-130a suppressed the expression of PPAR $\gamma$ by directly targeting its 3 'UTR. Additionally, the downregulation of miR-130a was inversely associated with tumor stage, metastasis, survival and the presence of the tumor macrophage marker CD163, in NSCLC samples.

\section{Materials and methods}

Cell lines. The human acute monocyte THP-1 leukemia cell line was purchased from the Institute of Biochemistry and Cell Biology, Chinese Academy of Sciences (Shanghai, China). To generate macrophage-like differentiated THP-1 cells, THP-1 cells were seeded in tissue culture flasks at $3 \times 10^{6}$ cells/flask and exposed to $320 \mathrm{nM}$ PMA in the culture medium for $48 \mathrm{~h}$ as previously described (21). Following incubation, the PMA-containing medium was removed and adherent (differentiated) cells were incubated in fresh culture medium for subsequent experiments. To generate M1-polarized THP-1 macrophages, dTHP-1 cells were cultured with IFN- $\gamma$ (100 U/ml) for another $48 \mathrm{~h}$. To generate M2-polarized THP-1 macrophages, the dTHP-1 cells were cultured with M-CSF (100 ng/ml) for an additional $48 \mathrm{~h}$.

Tissue specimens. From December 2010 to November 2014, a total of 75 NSCLC adenocarcinoma specimens and 75 matched normal tissue from adjacent regions were collected from the patients undergoing curative resection and diagnosed histopathologically at the Department of Medical Oncology, Cancer Hospital Institute, Chinese Academy of Medical Science, Peking Union Medical College (Beijing, China). The samples were immediately frozen and stored in liquid nitrogen prior to analysis. None of the patients received chemotherapy or radiotherapy prior to the surgical excision. All the patients provided informed consent for the sample collection. The procedure was approved and supervised by the Institutional Review Board (IRB) of the Cancer Institute/Hospital of
Chinese Academy of Medical Sciences and Peking Union Medical College.

Quantitative PCR. RNA was isolated using TRIzol (Invitrogen Life Technologies, Carlsbad, CA, USA), RNeasy (Qiagen, Hilden, Germany), or miR-Neasy (Qiagen) as per the manufacturer's instructions. The synthesis of cDNA was performed with $0.5 \mu \mathrm{g}$ RNA using PrimeScript ${ }^{\circledR}$ RT Master Mix (Perfect Real-Time) (Takara Bio Inc., Dalian, China). RT-qPCR analysis was performed in triplicate on LightCycler ${ }^{\circledR} 480$ II (Roche Applied Science Indianapolis, IN, USA) using SYBR ${ }^{\circledR}$ Premix Ex Taq ${ }^{\mathrm{TM}}$ (Perfect Real-Time) (Takara Bio Inc.) and the results were normalized according to the expression levels of GAPDH RNA. Results were expressed using the $2^{-\Delta \Delta C T}$ methods. The primers for the selected genes are shown in Table I.

Western blotting. Western blot analysis was performed as previously described (22). Briefly, the cells were lysed in cell lysis buffer [1\% NP-40, $20 \mathrm{mM}$ Tris- $\mathrm{HCl}(\mathrm{pH} 7.6)$, $0.15 \mathrm{M} \mathrm{NaCl}, 3 \mathrm{mM}$ EDTA, $3 \mathrm{mM}$ ethylene glycol tetraacetic acid (EGTA), $1 \mathrm{mM}$ phenylmethylsulfonyl fluoride, $2 \mathrm{mM}$ sodium vanadate, $20 \mathrm{mg} / \mathrm{ml}$ aprotinin and $5 \mathrm{mg} / \mathrm{ml}$ leupeptin]. Following treatment, the lysates were purified by centrifugation and denatured by boiling in loading buffer. Equal amounts of protein samples were separated on $10 \%$ sodium dodecyl sulfate polyacrylamide gel electrophoresis (SDS-PAGE), and electrophoretically transferred to a nitrocellulose membrane. Following blocking with 5\% non-fat milk at room temperature for $1.5 \mathrm{~h}$, the membrane was incubated with rabbit anti-human mono-clonal primary antibody with an appropriate dilution of antibodies (1:1,000-1:2,000) overnight at $4^{\circ} \mathrm{C}$ and then with a horseradish peroxidaseconjugated goat anti-rabbit secondary antibody at 1:5,000 dilution for $1 \mathrm{~h}$ at room temperature, and detected using the Western Lightning Chemiluminescent detection reagent (Amersham, Freiburg, Germany).

Luciferase activity assay. To construct pGL3-PPAR $\gamma$-3'UTR, the full length $3^{\prime} \mathrm{UTR}$ of the human PPAR $\gamma$ mRNA was amplified by PCR and cloned into the pGL3-control vector (Promega, Madison, WI, USA). For the reporter assays, 293T cells were transiently transfected with reporter plasmid and 
miR-130a mimic, using Lipofectamine 2000 (Invitrogen Life Technologies). Reporter assays were performed $48 \mathrm{~h}$ post-transfection using the dual-luciferase assay system (Promega), normalized for transfection efficiency by co-transfected Renilla luciferase.

Flow cytometry. The samples were incubated with PE-CD86 and FITC-CD206 (BioLegend, San Diego, CA, USA) according to the manufacturers' instructions. Fluorescent conjugated with Alexa Fluor 488 (Invitrogen Life Technologies) was used as a secondary antibody. For each sample at least $1 \times 10^{4}$ cells were analyzed.

ELISA. Cytokine concentrations in the culture supernatants were determined by ELISA kits according to the manufacturer's instructions (eBioscience, San Diego, CA, USA).

Bioinformatics. Prediction of putative miR-130a targets was performed by using the online software, TargetScan (http://www.targetscan.org/) in conjunction with miRanda (http://www.microrna.org/microrna/home.do) and PicTar (http://pictar.mdc-berlin.de/). MiRanda was used for the primary screening of miRNA target sites with cut-off values for free energy $(\Delta G) \leq 14 \mathrm{kcal} / \mathrm{mole}$ and scores $>70$. PicTar and TargetScan is an algorithm for the identification of miRNA targets.

Statistical analysis. Data are presented as mean \pm SD. One-way ANOVA followed by the Bonferroni test was performed for multiple group comparisons. The Student's t-test was used for a comparison between two groups. Pearson's correlation was used to analyze the relationship between the expression of miR-130a and CD163 and PPAR $\gamma$ mRNA. $P<0.05$ was considered to indicate a statistically significant result.

\section{Results}

M1 macrophages demonstrate greater expression of miR-130a compared to M2 macrophages. We investigated the levels of miR-130a in the proinflammatory M1 subset and immunosuppressive M2 subset. These subsets were induced in vitro and characterized based on their phenotypic characteristics. As shown in Fig. 1A, the M2 macrophages expressed lower levels of TNF- $\alpha$, IL-1 $\beta$ and iNOS compared to the M1 macrophages but higher levels of CD163, CCL17 and CCL22 mRNA. Furthermore, the shift in macrophages was detected using ELISA analysis. As shown in Fig. 1B, M1 enhanced the levels of TNF- $\alpha$ and IL-1 $\beta$, whereas M2 reduced the levels of CCL17 and CCL22.

The expression of miR-130a was detected by RT-qPCR. We found that M1 macrophages exhibited a considerably higher level of miR-130a compared to the M2 macrophages (Fig. 1C). The initial findings suggested that miR-130a participates in macrophage polarization. To examine whether miR-130a contributes to the plasticity of macrophage polarization, we converted one population into another by culturing M1 macrophages with M-CSF and M2 macrophages with IFN- $\gamma$. As shown in Fig. 1D, M1 to M2 macrophages conversion resulted in decreased miR-130a, whereas M2 to M1 conversion led to increased miR-130a expression.
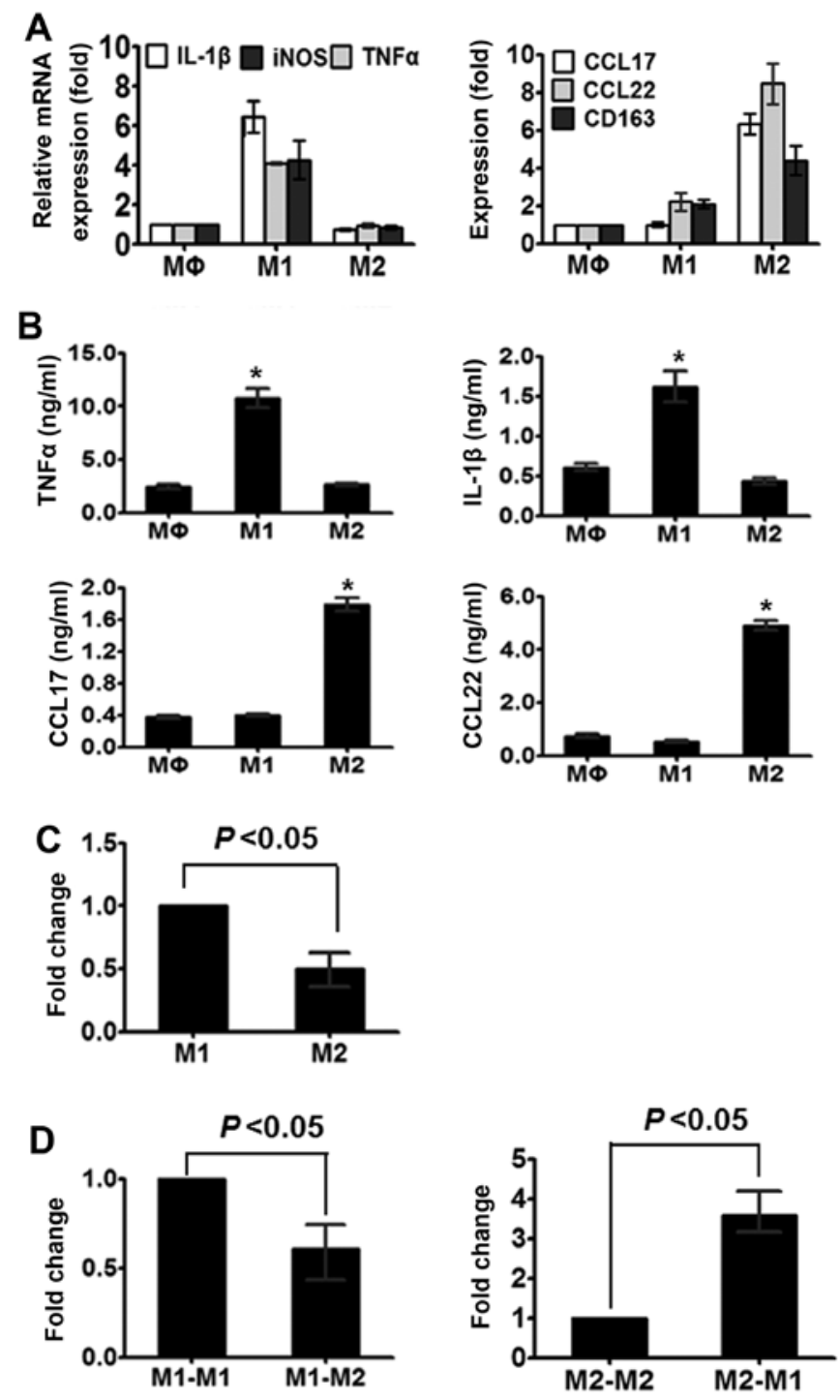

Figure 1. M1 shows a greater expression of miR-130a compared to M2. THP-1 cells were cultured in IFN- $\gamma(100 \mathrm{U} / \mathrm{ml})$ or M-CSF $(100 \mathrm{ng} / \mathrm{ml})$ for $48 \mathrm{~h}$ to establish M1 and M2 macrophages. (A) RNA was isolated and levels of M1 and M2-associated makers determined by RT-qPCR. (B) Protein expressions of M1 and M2-associated makers was detected by ELISA method. $n=3$, mean \pm SD. ${ }^{*} \mathrm{P}<0.05$ compared with $\mathrm{M} \phi$ macrophages. (C) RNA was isolated and levels of miR-130a determined by RT-qPCR. U6 snRNA was used as an internal control. (D) M1 and M2 macrophages were established as in (A). The cells were then cultured in fresh media containing M-CSF or IFN- $\gamma$ for two more days to induce the transition from M1 to M2 or vice versa. RNA was isolated and levels of miR-130a determined. $n=3$, mean \pm SD.

Overexpression of miR-130a reduces M2-polarized THP-1 macrophages. To determine whether miR-130a participates in macrophage polarization, we transfected M2 macrophage, which have lower levels of miR-130a compared to M1 macrophages. We found that the overexpression of miR-130a with miR-130a mimics (Fig. 2A) in M2 macrophages enhanced the mRNA and protein levels of TNF- $\alpha$ and iNOS, and reduced the expression of IL-10 and CCL22 (Fig. 2B and C). Additionally, the flow cytometric analysis revealed that miR-130a overexpression significantly enhanced CD80 (M1 marker), but inhibited the expression of CD206 (M2 marker) on M2 macrophages (Fig. 2D). 

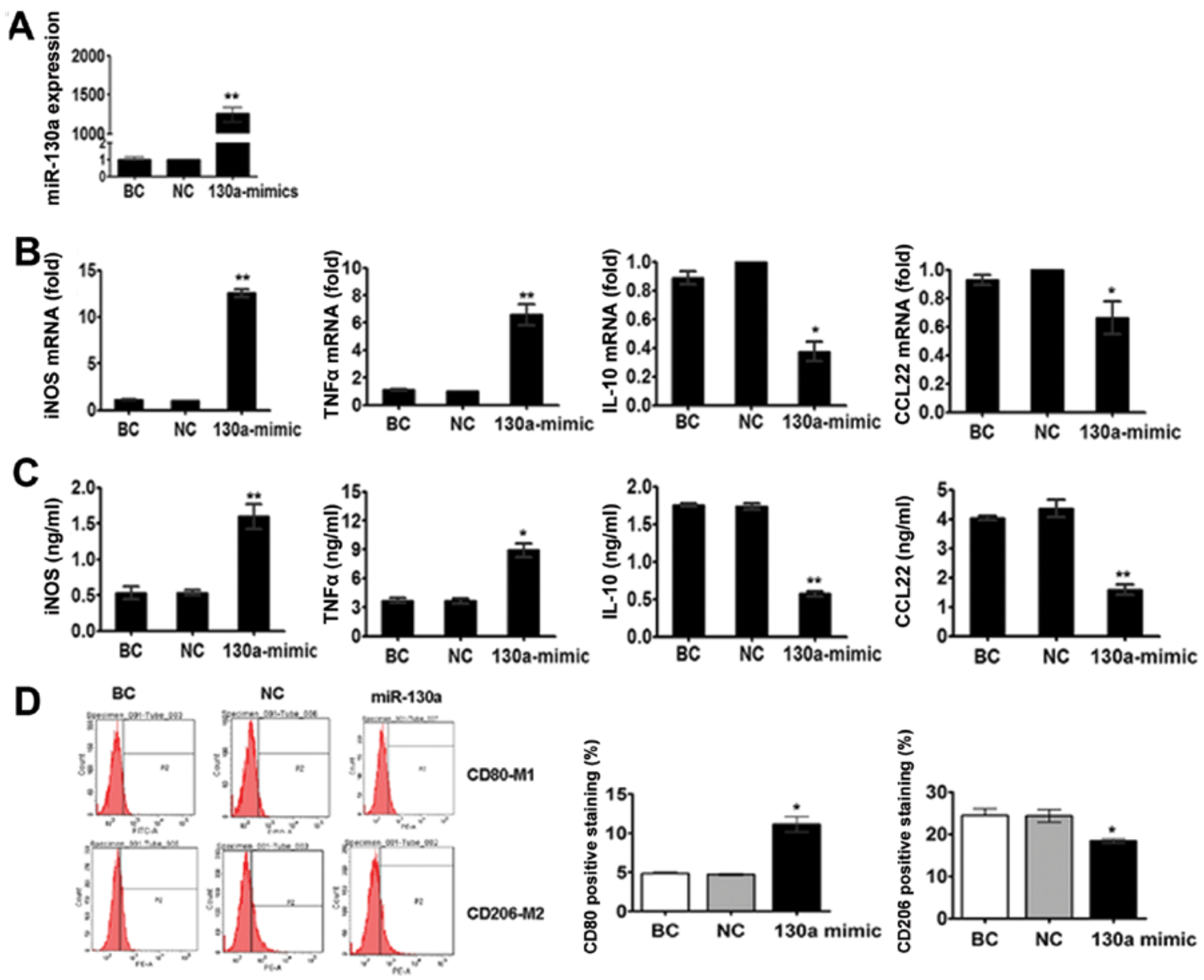

Figure 2. Overexpression of miR-130a reduces M2-polarized THP-1 macrophages. M2 macrophages were transfected with $20 \mathrm{nM}$ control mimics or miR130a mimics. Forty-eight hours after transfection, the cells were harvested and RNA and protein isolated. (A) RT-qPCR showing the relative expression of miR-130a in untreated M2 macrophages (blank control, BC), and cells treated with miR-130a mimics and negative controls (NC). Relative (B) mRNA and (C) protein levels of proinflammatory cytokines were assayed by qPCR or ELISA, respectively. (D) Expression of CD80 and CD206 was detected by flow cytometry. Representative histograms and average relative mean positive percentage. The results are from at least three independent experiments and presented as mean $\pm \mathrm{SD}$. ${ }^{*} \mathrm{P}<0.05,{ }^{* *} \mathrm{P}<0.01$ vs. negative controls.
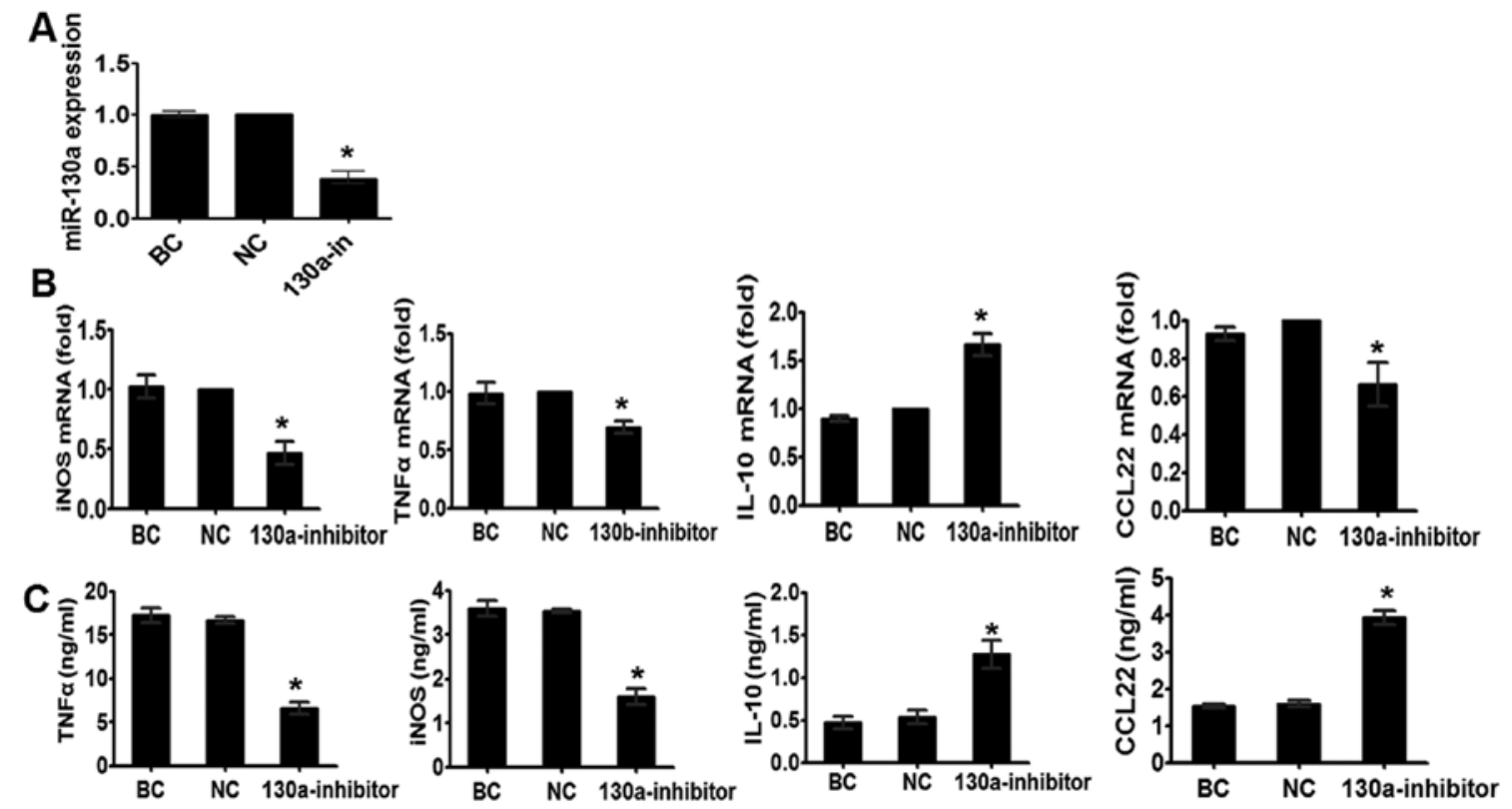

Figure 3. Inhibition of miR-130a results in M2 polarization of macrophages. (A) RT-qPCR showing the relative miR-181a expression in untreated M1 macrophages (BC), and cells treated with miR-130a inhibitors and the negative control (NC). Relative (B) mRNA and (C) protein levels of proinflammatory cytokines were assayed by qPCR or ELISA, respectively. The results are from at least three independent experiments and presented as mean \pm SD. " $\mathrm{P}<0.05$, ${ }^{* *} \mathrm{P}<0.01$ vs. negative controls. 
A

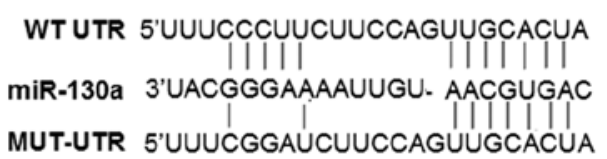

B

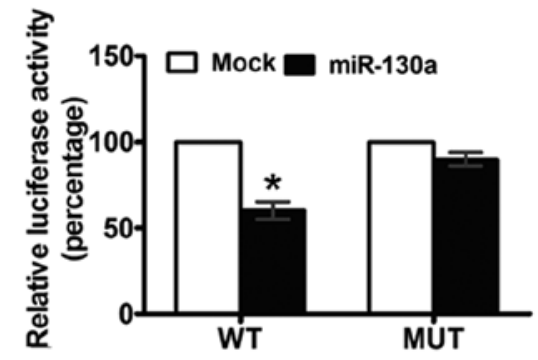

C

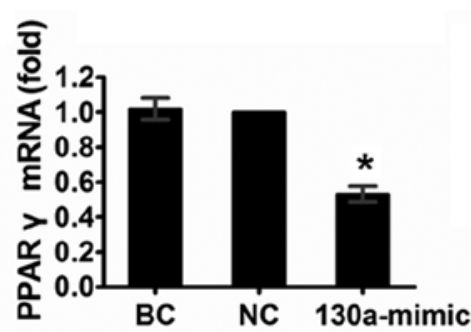

D
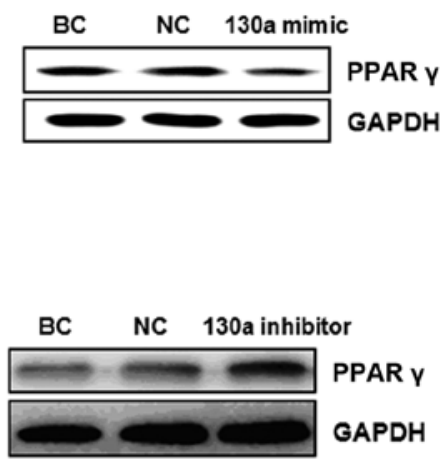
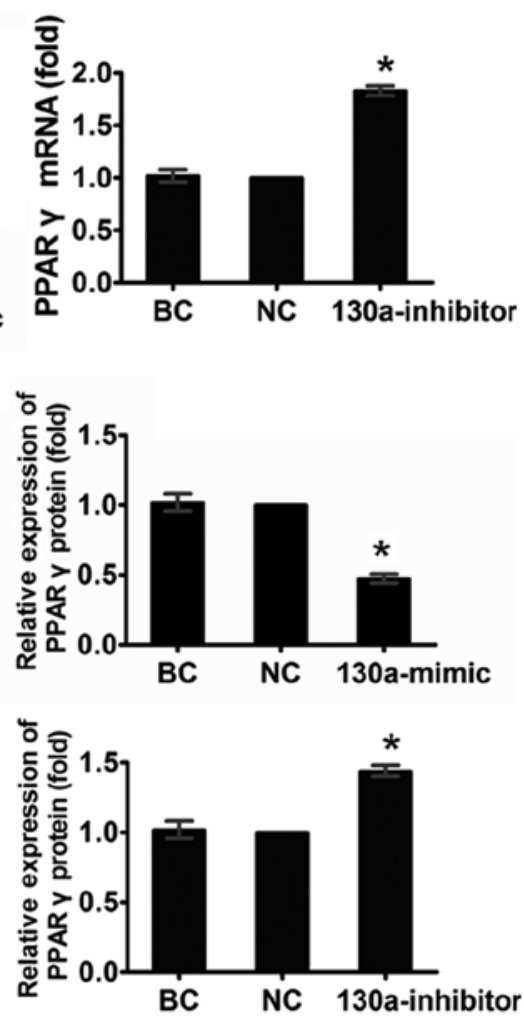

Figure 4. miR-130a inhibits the expression of PPAR $\gamma$ by targeting its 3'UTR. (A) Alignment of miRNA-130a with PPAR $\gamma$ 3'UTR targeted site (nucleotides 42-49 of human PPAR $\gamma$ 3'UTR). The mutations introduced into the luciferase reporter construct are shown as well. (B) Analysis of luciferase activity. Cells were co-transfected with pGL3-PPAR $\gamma$ 3'UTR and miR-130a mimics, firefly luciferase reporter containing either wild-type or mutant 3'UTR (indicated as WT or MUT on the x-axis), and Renilla luciferase expression construct (as an internal control). Luciferase activity was assayed $48 \mathrm{~h}$ after transfection. Firefly luciferase values, normalized for Renilla luciferase, are presented. " $\mathrm{P}<0.05$, compared with cells transfected with pGL3-control vector. (C) RT-qPCR and (D) western blot analysis were used to assay the expression level of PPAR $\gamma$ in transfected cells at $48 \mathrm{~h}$ post-transfection. THP-1 cells were transfected with $20 \mathrm{nM}$ of miR-130a mimic, miR-130a inhibitor or their controls for $48 \mathrm{~h}$. GAPDH served as an internal control. Three representative experiments are shown for $(C)$ and (D). The data represent the mean \pm SD of three independent experiments done in duplicates. ${ }^{*} \mathrm{P}<0.05$ vs. negative controls (NC).

Inhibition of miR-130a results in M2 polarization of macrophages. Earlier, we showed that miR-130a suppresses the expression of the M2 phenotype. Therefore, we determined whether miR-130a inhibition in M1 macrophages, which have higher levels of miR-130a compared to M2 macrophages, demonstrated an effect opposite to that observed in M2 macrophages transfected with miR-130a mimics. To verify this, we simulated the THP-1 cells with IFN- $\gamma$ for $48 \mathrm{~h}$. As shown in Fig. 3B and C, miR-130a knockdown decreased the IFN- $\gamma$ induced expression of TNF- $\alpha$ and iNOS, and enhanced the expression of M2-associated genes $I L-10$ and $C C L 22$. Given our findings that $\mathrm{M} 2$ macrophages with overexpression of miR-130a decreased the anti-inflammatory response to M-CSF, the results showed that miR-130a has a suppressive role in M2 macrophage polarization.

miR-130a inhibits the expression of PPAR $\gamma$ by targeting its $3^{\prime} U T R$. To elucidate the molecular mechanism by which miR-130a modulates the macrophage polarization, we predicted the targets of miR-130a by using bioinformatics tools TargetScan, PicTar and miRanda. The programs predicted PPAR $\gamma$ as a target of miR-130a, and one potential miR-130a target sites at thepositions $42 \mathrm{nt}$ in the PPAR $\gamma$
3'UTR was identified (Fig. 4A). To verify that PPAR $\gamma$ is a functional target of miR-130a, we cloned a reporter plasmid containing the wide-type $3^{\prime} \mathrm{UTR}$ of PPAR $\gamma$ at the $3^{\prime}$ position of the firefly luciferase reporter gene. In parallel, we constructed reporter plasmids in which the observed target sequences were mutated individually or in combination, and transfected 293T cells with these constructs with miR-130a mimic and NC. Luciferase activity was markedly reduced in cells transfected with miR-130a mimics and wild-type PPAR $\gamma-3$ 'UTR reporter plasmid-transfected cells, compared to the cells transfected with NC mimics, but had no effect on the mutant 3'UTR of PPAR $\gamma$ (Fig. 4B), indicating that miR-130a can regulate gene expression through the putative binding site in the 3'UTR of PPAR $\gamma$ mRNA.

To confirm that miR-130a represses PPAR $\gamma$ expression in THP-1 cells, we performed RT-qPCR analysis and found that the transfection of miR-130a mimic led to a significant decrease in the PPAR $\gamma$ mRNA level, while the transfection of miR-130a inhibitor led to a significant increase in the PPAR $\gamma$ mRNA level compared to the respective controls (Fig. 4C). In addition, western blot analysis showed that transfection of the miR-130a mimic led to a significant decrease in the PPAR $\gamma$ protein level (Fig. 4D) and miR-130a inhibitor resulted in an 
Table I. Association of miR-130a expression with clinicopathological characteristics of NSCLC.

\begin{tabular}{|c|c|c|c|c|c|}
\hline \multirow[b]{2}{*}{ Characteristics } & \multirow[b]{2}{*}{ No. } & \multicolumn{2}{|c|}{ Expression of miR-130a ${ }^{a}$} & \multirow[b]{2}{*}{$\chi^{2}$} & \multirow[b]{2}{*}{ P-value } \\
\hline & & Low & High & & \\
\hline Age (years) & & & & 1.335 & 0.248 \\
\hline$\leq 57$ & 50 & $19(38.0)$ & $31(62.0)$ & & \\
\hline$>57$ & 25 & $13(52.0)$ & $12(48.0)$ & & \\
\hline Gender & & & & 0.819 & 0.366 \\
\hline Male & 40 & $19(47.5)$ & $21(52.5)$ & & \\
\hline Female & 35 & $13(37.1)$ & $22(62.9)$ & & \\
\hline Smoking history (years) & & & & 0.054 & 0.817 \\
\hline$\leq 10$ & 41 & $17(41.5)$ & $24(58.5)$ & & \\
\hline$>10$ & 34 & $15(44.1)$ & $19(55.9)$ & & \\
\hline Metastasis & & & & 6.141 & 0.013 \\
\hline No & 45 & $14(31.1)$ & $31(68.9)$ & & \\
\hline Yes & 30 & $18(60.0)$ & $12(40.0)$ & & \\
\hline Histological subtype & & & & 2.252 & 0.133 \\
\hline Adenocarcinoma & 38 & $13(34.2)$ & $25(65.8)$ & & \\
\hline Squamous carcinoma & 37 & $19(51.4)$ & $18(48.6)$ & & \\
\hline TNM stage & & & & 8.715 & 0.003 \\
\hline $\mathrm{I}+\mathrm{II}$ & 31 & $7(22.6)$ & $24(77.4)$ & & \\
\hline III+IV & 44 & $25(56.8)$ & $19(43.2)$ & & \\
\hline
\end{tabular}

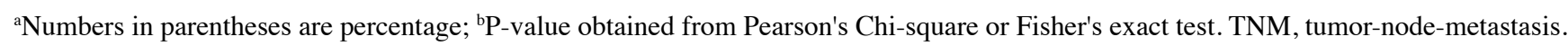
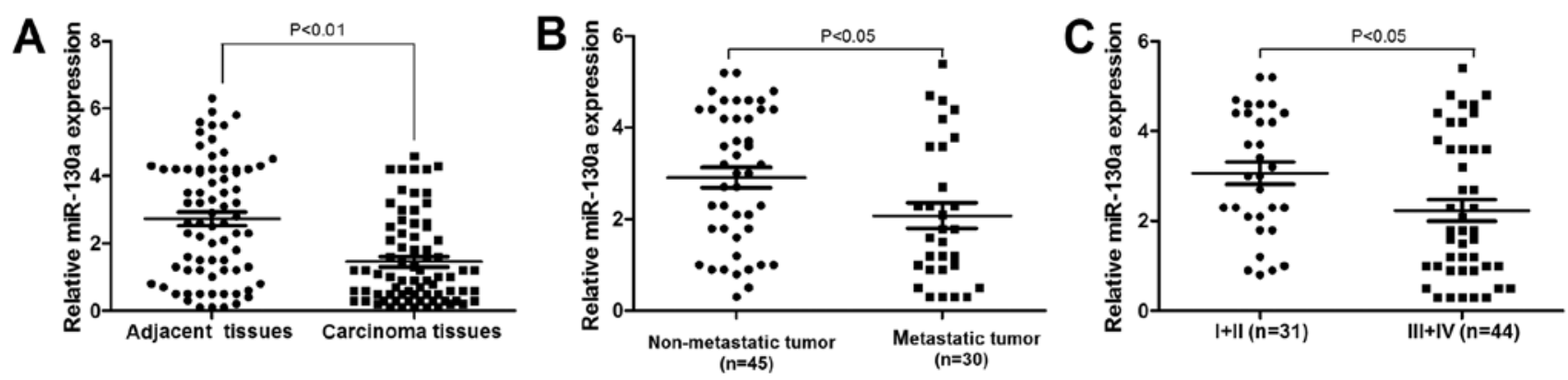

Figure 5. Downregulation of miR-130a is inversely associated with advanced stage and lymph node metastasis of NSCLC. qPCR analysis of miR-130a expression in 75 paired cancerous tissues $(\mathrm{T})$ and their adjacent non-cancerous lung tissues (ANT). Transcript levels were normalized to U6 expression (P<0.01). (A) miR-130a expression level is significantly lower in NSCLC tissues than in their matched normal tissues. (B) Low-level expression of miR-130a is associated with lymph node metastasis of NSCLC $(\mathrm{P}<0.05)(\mathrm{C})$. Low-level expression of miR-130a is associated with high tumor stage of NSCLC $(\mathrm{P}<0.05)$.

increased protein expression of PPAR $\gamma$. Taken together, these results provide evidence that miR-130a inhibits the expression of PPAR $\gamma$ by directly targeting the 3'UTR of PPAR $\gamma$.

Expression of miR-130a is inversely associated with advanced stage and lymph node metastasis of NSCLC. We examined miR-130a expression in 75 NSCLC patients samples. miR-130a was significantly downregulated in NSCLC tissues, compared to that in non-tumor tissues ( $\mathrm{P}<0.05$, Fig. $5 \mathrm{~A})$. We also investigated the association between miR-130a expression and established clinicopathological characteristics. As indicated in Table I, miR-130a was significantly associated with the metastasis and TNM of NSCLC, and the expression level of miR-130a in tumor tissues decreased statistically with the increasing stage of NSCLC $(\mathrm{P}<0.05)$ (Fig. 5B). In addition, miR-130a expression was significantly reduced in NSCLC, exhibiting lymph node metastasis compared to NSCLC that did not exhibit lymph node metastasis (Fig. 5C). No significant association to age, gender, smoking history or histological subtype was identified. Therefore, the low miR-130a expression was closely associated with the progression and metastasis of NSCLC.

miR-130a downregulation predicts poor overall survival in NSCLC patients. To evaluate the potential clinical relevance of the downregulated miR-130a with regard to prognosis, the Kaplan-Meier survival analysis was performed using overall 


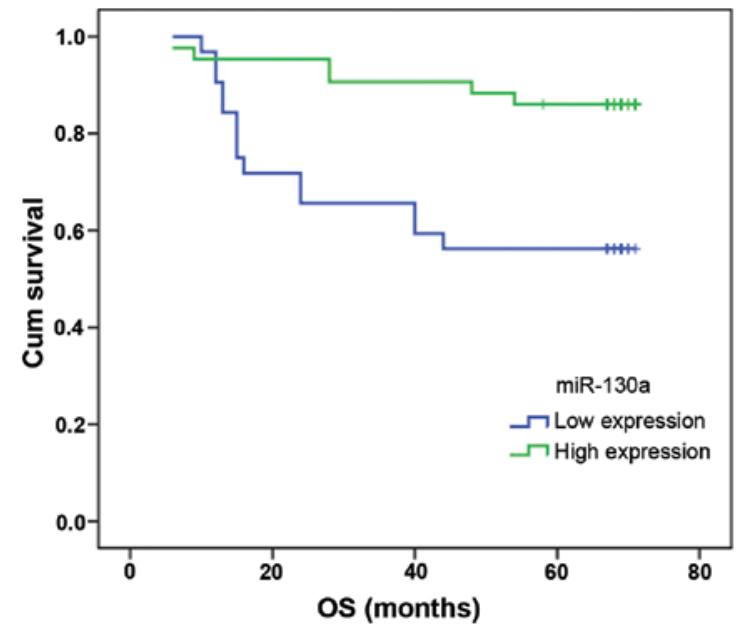

Figure 6. Recurrence-free survival among NSCLC patients according to miR-130a expression. Overall survival curves for two groups defined by a low and high expression of miR-130a in patients with NSCLC.
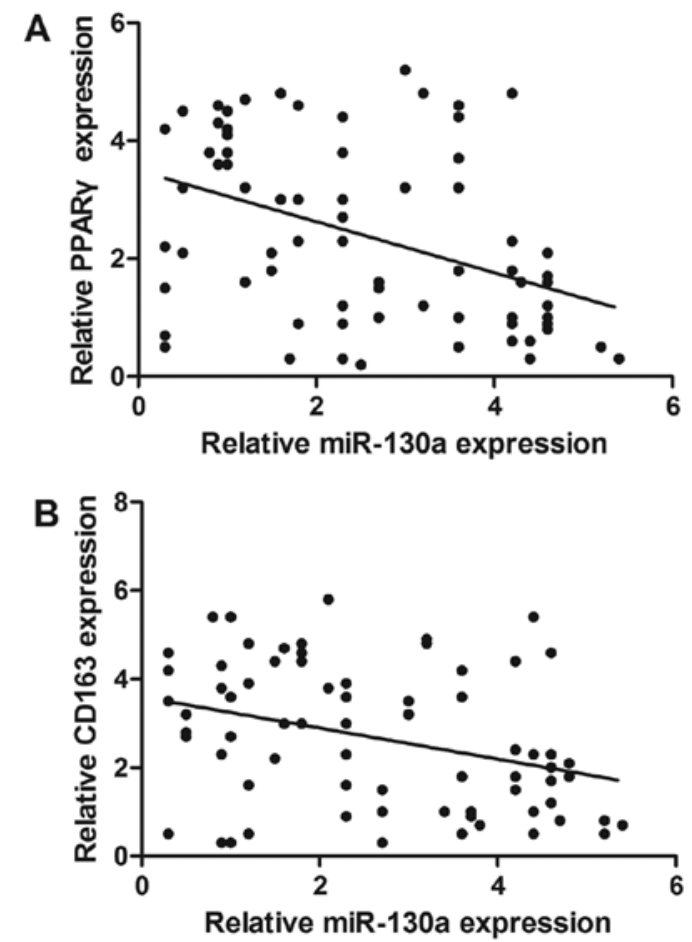

Figure 7. miR-130a was inversely correlated with CD163 and PPAR $\gamma$ expression in NSCLC tissues. Expression of miR-130a, CD163 and PPAR $\gamma$ in 75 NSCLC tissues were detected by RT-qPCR. (A) Correlation analysis between miR-130a and CD163 mRNA level in NSCLC tissues (Spearman's correlation analysis, $r=-0.340 ; \mathrm{P}<0.01$ ). (B) Correlation analysis between miR-130a and PPAR $\gamma$ mRNA level in NSCLC tissues (Spearman's correlation analysis, $\mathrm{r}=-0.426 ; \mathrm{P}<0.01)$.

survival. The results indicated that miR-130a was significantly associated with patient survival (Fig. 6). Patients with a high fold change of miR-130a survived longer $(n=43$; median survival of 65 months) than the patients with a low fold change of miR-130a ( $\mathrm{n}=32$; median survival of 49 months) $(\mathrm{P}=0.003)$.

miR-130a expression is associated with CD163 and PPAR $\gamma$ expression in NSCLC tissues. We investigated the association between miR-130a and CD163, an M2 macrophage marker. As shown in Fig. 7, the Spearman's correlation analysis revealed a direct correlation between CD163 and miR-130a. The lower CD163 mRNA expression tumors had a substantially higher miR-130a content compared to the reduction in miR-130a levels with increasing CD163 macrophage contents indicating an inverse association between miR-130a expression and macrophages. In addition, the inverse association was observed between PPAR $\gamma$ mRNA expression and miR-130a level.

\section{Discussion}

One of the hallmarks of malignancy is the polarization of TAMs from a pro-immune (M1-like) phenotype to an immun0suppressive (M2-like) phenotype. The two distinct subsets, which coexist in tumors, adapt to the changing tumor microenvironment, and can be re-educated by immunoregulatory cues $(23,24)$. This event has primed interest in developing therapies, with the aim of skewing TAMs to an M1-like phenotype (25). Nonetheless, only a few molecules have been identified to orchestrate this process thus far. Evidence has shown that miRNAs are relevant in macrophage activation and function. For example, miR-155, -146, -147, -9 and -21 are induced by TLR ligands $(26,27)$. However, the potential of miRNAs to alter macrophage phenotype and function has been rarely studied. Our investigation provides insight into the role of miR-130a in the control of macrophage polarization.

The biological role of miR-130a in the macrophage has yet to be reported. In the present study, we demonstrate that miR-130a is at a higher level (M1 macrophages) comapred to M2 macrophages. The transfection of macrophages with miR-130a mimic resulted in the downregulation of markers and cytokines associated with the phenotype of the classically activated (M2) macrophages CD206, IL-10 and CCL22, whereas cytokines and markers associated with the phenotype of alternatively activated (M1) regulatory macrophages CD80, iNOS and TNF- $\alpha$, were upregulated. These results suggest that miR-130a skews their polarization from an M2 towards an M1 phenotype. Our results therefore reveal a critical role of miR-130a in the induction of pathogenic M1 macrophage activation and the transition between the pro-and anti-inflammatory phenotypes, which is believed to provide novel insight into the molecular regulation of the functional shaping of macrophages and associated inflammatory disorders.

PPAR $\gamma$ is a ligand-activated transcription factor belonging to the nuclear receptor superfamily. It plays a pivotal role in the control of lipid metabolism and maintenance of energetic homeostasis. PPAR $\gamma$ has been known to inhibit pro-inflammatory gene expression through several mechanisms, including the transrepression of NF- $\mathrm{KB}$ (28). For macrophage programming towards M2 polarization, the activation of PPAR $\gamma$ is considered to be critical (29). It has been demonstrated that IL-4 and IL-13 induce the expression and activation of PPAR $\gamma(30,31)$. The present study provides evidence that miR-130a regulates inflammatory cytokine production via PPAR $\gamma$ targeting. The results of four sets of experiments from the present study support this conclusion. First, the bioinformatics analysis reveal that PPAR $\gamma$ is a potential target of miR-130a. Second, the results from the luciferase reporter 
assay demonstrate that miR-130a may regulate PPAR $\gamma$ protein expression through the conserved miR-130a binding site in the 3'UTR of the PPAR $\gamma$ mRNA. Third, the level of PPAR $\gamma$ protein is downregulated by the ectopic expression of miR-130a, but is upregulated by the inhibition of endogenous miR-130a with the synthetic inhibitor. Fourth, an inverse association was observed between PPAR $\gamma$ mRNA expression and the miR-130a level in NSCLC tissues. These results clearly indicate that PPAR $\gamma$ is a target for miR-130a and that miR-130a controls cytokine production in THP-1 cells by releasing its translational inhibition of PPAR $\gamma$.

TAMs are abundant components of NSCLC and play a key role in the progression of NSCLC (32). Aberrant miRNAs expressions have been observed in different types of cancer and their expression signatures can be extremely informative for the diagnosis of cancer (33-35). The above results show that miR-130a is a key factor in M1/M2 modulation, raising the question of whether the evaluation of miR-130a expression has a prognostic role in NSCLC patients. Therefore, we examined the role of miR-130a in NSCLC. To the best of our knowledge, we report for the first time that miR-130a expression was downregulated in NSCLC samples compared with the adjacent tissues. Tumors with low miR-130a levels were associated with high tumor stage and poor recurrence-free survival suggesting that miR-130a is a potential marker for tumor progression. Therefore, miR-130a may be a novel tumor-suppressor miRNA, and its downregulation may contribute to lung cancer progression and metastasis. Few reports have shown the involvement of miR-130a in tumorigenesis. Pan et al (36) have demonstrated that mRNA-130a inhibits cell proliferation, invasion and migration in human breast cancer by targeting the RAB5A. Chen et al (37) have reported that miR-130a can predict response to temozolomide in patients with glioblastoma multiforme. Acunzo et al (38) showed that miR-130a targets MET and induces TRAIL sensitivity in NSCLC by downregulating miR-221 and -222 . In addition to the link to tumor prognosis, miR-130a was strongly and inversely correlated with CD163 expression. CD163, a marker of M2 macrophages, has been studied in several aggressive tumors, and the increased expression of CD163 was significantly associated with a poor overall survival in various types of cancer (39-41). Our results are in accordance with those obtained from THP-1 cells, suggesting that miR-130a are important factors in macrophage polarization.

In conclusion, in the present study, we have identified an unknown role for miR-130a in macrophages, providing further insight into the complexities of macrophage plasticity, suggesting that targeting miR-130a may have unforeseen effects on macrophage function. Additionally, miR-130a is frequently downregulated in NSCLC and correlates with tumor stage and poorer patients' prognosis. These results suggest that miR-130a functions as a tumor suppressor in NSCLC and is a potential molecular target for NSCLC therapy.

\section{References}

1. Torre LA, Bray F, Siegel RL, Ferlay J, Lortet-Tieulent J and Jemal A: Global cancer statistics, 2012. CA Cancer J Clin 65: 87-108, 2015.

2. Byers LA and Rudin CM: Small cell lung cancer: Where do we go from here? Cancer 121: 664-672, 2015.
3. Murray PJ, Allen JE, Biswas SK, Fisher EA, Gilroy DW, Goerdt S, Gordon S, Hamilton JA, Ivashkiv LB, Lawrence T, et al: Macrophage activation and polarization: Nomenclature and experimental guidelines. Immunity 41: 14-20, 2014.

4. Cook J and Hagemann T: Tumour-associated macrophages and cancer. Curr Opin Pharmacol 13: 595-601, 2013.

5. Gordon S: Alternative activation of macrophages. Nat Rev Immunol 3: 23-35, 2003.

6. Ruffell B and Coussens LM: Macrophages and therapeutic resistance in cancer. Cancer Cell 27: 462-472, 2015

7. Krausgruber T, Blazek K, Smallie T, Alzabin S, Lockstone $H$, Sahgal N, Hussell T, Feldmann M and Udalova IA: IRF5 promotes inflammatory macrophage polarization and TH1-TH17 responses. Nat Immunol 12: 231-238, 2011.

8. Allavena P, Sica A, Solinas G, Porta C and Mantovani A: The inflammatory micro-environment in tumor progression: The role of tumor-associated macrophages. Crit Rev Oncol Hematol 66: $1-9,2008$

9. Sica A and Mantovani A: Macrophage plasticity and polarization: In vivo veritas. J Clin Invest 122: 787-795, 2012.

10. Santoni M, Massari F, Amantini C, Nabissi M, Maines F, Burattini L, Berardi R, Santoni G, Montironi R, Tortora G, et al: Emerging role of tumor-associated macrophages as therapeutic targets in patients with metastatic renal cell carcinoma. Cancer Immunol Immunother 62: 1757-1768, 2013.

11. Ruhrberg C and De Palma M: A double agent in cancer: Deciphering macrophage roles in human tumors. Nat Med 16: 861-862, 2010.

12. Chen P and Bonaldo P: Role of macrophage polarization in tumor angiogenesis and vessel normalization: Implications for new anticancer therapies. Int Rev Cell Mol Biol 301: 1-35, 2013.

13. Ohri CM, Shikotra A, Green RH, Waller DA and Bradding P: Macrophages within NSCLC tumour islets are predominantly of a cytotoxic M1 phenotype associated with extended survival. Eur Respir J 33: 118-126, 2009.

14. Zhang J, Cao J, Ma S, Dong R, Meng W, Ying M, Weng Q, Chen Z, Ma J, Fang Q, et al: Tumor hypoxia enhances non-small cell lung cancer metastasis by selectively promoting macrophage M2 polarization through the activation of ERK signaling. Oncotarget 5: 9664-9677, 2014.

15. Sun J, Mao Y, Zhang YQ, Guo YD, Mu CY, Fu FQ and Zhang XG: Clinical significance of the induction of macrophage differentiation by the costimulatory molecule $\mathrm{B} 7-\mathrm{H} 3$ in human non-small cell lung cancer. Oncol Lett 6: 1253-1260, 2013.

16. Domagala-Kulawik J: The role of the immune system in non-small cell lung carcinoma and potential for therapeutic intervention. Transl Lung Cancer Res 4: 177-190, 2015.

17. Bartel DP: MicroRNAs: Target recognition and regulatory functions. Cell 136: 215-233, 2009.

18. Hammond SM: An overview of microRNAs. Adv Drug Deliv Rev 87: 3-14, 2015.

19. Lan H, Lu H, Wang $X$ and Jin H: MicroRNAs as potential biomarkers in cancer: opportunities and challenges. Biomed Res Int 2015: 125094, 2015.

20. Jasinski-Bergner S, Mandelboim O and Seliger B: The role of microRNAs in the control of innate immune response in cancer. J Natl Cancer Inst 106: dju257, 2014.

21. Park EK, Jung HS, Yang HI, Yoo MC, Kim C and Kim KS: Optimized THP-1 differentiation is required for the detection of responses to weak stimuli. Inflamm Res 56: 45-50, 2007.

22. Wang XF, Wang HS, Wang H, Zhang F, Wang KF, Guo Q, Zhang G, Cai SH and Du J: The role of indoleamine 2,3-dioxygenase (IDO) in immune tolerance: Focus on macrophage polarization of THP-1 cells. Cell Immunol 289: 42-48, 2014.

23. Movahedi K, Laoui D, Gysemans C, Baeten M, Stangé G, Van den Bossche J, Mack M, Pipeleers D, In't Veld P, De Baetselier P, et al: Different tumor microenvironments contain functionally distinct subsets of macrophages derived from Ly6C(high) monocytes. Cancer Res 70: 5728-5739, 2010.

24. Pucci F, Venneri MA, Biziato D, Nonis A, Moi D, Sica A, Di Serio C, Naldini L and De Palma M: A distinguishing gene signature shared by tumor-infiltrating Tie2-expressing monocytes, blood 'resident' monocytes, and embryonic macrophages suggests common functions and developmental relationships. Blood 114: 901-914, 2009.

25. Rolny C, Mazzone M, Tugues S, Laoui D, Johansson I, Coulon C, Squadrito ML, Segura I, Li X, Knevels E, et al: HRG inhibits tumor growth and metastasis by inducing macrophage polarization and vessel normalization through downregulation of PIGF. Cancer Cell 19: 31-44, 2011. 
26. Alam MM and O'Neill LA: MicroRNAs and the resolution phase of inflammation in macrophages. Eur J Immunol 41: 2482-2485, 2011.

27. He M, Xu Z, Ding T, Kuang DM and Zheng L: MicroRNA-155 regulates inflammatory cytokine production in tumor-associated macrophages via targeting $\mathrm{C} / \mathrm{EBPbeta}$. Cell Mol Immunol 6: 343-352, 2009.

28. Ricote M, Li AC, Willson TM, Kelly CJ and Glass CK: The peroxisome proliferator-activated receptor-gamma is a negative regulator of macrophage activation. Nature 391: 79-82, 1998

29. Bouhlel MA, Derudas B, Rigamonti E, Dièvart R, Brozek J, Haulon S, Zawadzki C, Jude B, Torpier G, Marx N, et al: PPARgamma activation primes human monocytes into alternative M2 macrophages with anti-inflammatory properties. Cell Metab 6: 137-143, 2007.

30. Huang JT, Welch JS, Ricote M, Binder CJ, Willson TM, Kelly C, Witztum JL, Funk CD, Conrad D and Glass CK: Interleukin-4dependent production of PPAR-gamma ligands in macrophages by 12/15-lipoxygenase. Nature 400: 378-382, 1999.

31. Berry A, Balard P, Coste A, Olagnier D, Lagane C, Authier H, Benoit-Vical F, Lepert JC, Séguéla JP, Magnaval JF, et al: IL-13 induces expression of CD36 in human monocytes through PPARgamma activation. Eur J Immunol 37: 1642-1652, 2007.

32. Becker M, Müller CB, De Bastiani MA and Klamt F: The prognostic impact of tumor-associated macrophages and intra-tumoral apoptosis in non-small cell lung cancer. Histol Histopathol 29: 21-31, 2014.

33. Xue Z, Wen J, Chu X and Xue X: A microRNA gene signature for identification of lung cancer. Surg Oncol 23: 126-131, 2014

34. Wali RK, Hensing TA, Ray DW, Dela Cruz M, Tiwari AK, Radosevich A, Jepeal L, Fernando HC, Litle VR, Charlot M, et al: Buccal microRNA dysregulation in lung field carcinogenesis: Gender-specific implications. Int J Oncol 45: 1209-1215, 2014.
35. Piva R, Spandidos DA and Gambari R: From microRNA functions to microRNA therapeutics: Novel targets and novel drugs in breast cancer research and treatment (Review). Int J Oncol 43: 985-994, 2013.

36. Pan Y, Wang R, Zhang F, Chen Y, Lv Q, Long G and Yang K: MicroRNA-130a inhibits cell proliferation, invasion and migration in human breast cancer by targeting the RAB5A. Int J Clin Exp Pathol 8: 384-393, 2015.

37. Chen $\mathrm{H}, \mathrm{Li} X, \mathrm{Li} W$ and Zheng H: miR-130a can predict response to temozolomide in patients with glioblastoma multiforme, independently of O6-methylguanine-DNA methyltransferase. J Transl Med 13: 69, 2015.

38. Acunzo M, Visone R, Romano G, Veronese A, Lovat F, Palmieri D, Bottoni A, Garofalo M, Gasparini P, Condorelli G, et al: miR-130a targets MET and induces TRAIL-sensitivity in NSCLC by downregulating miR-221 and 222. Oncogene 31: 634-642, 2012.

39. Maniecki MB, Etzerodt A, Ulhøi BP, Steiniche T, Borre M, Dyrskjøt L, Orntoft TF, Moestrup SK and Møller HJ: Tumor-promoting macrophages induce the expression of the macrophage-specific receptor CD163 in malignant cells. Int J Cancer 131: 2320-2331, 2012.

40. He KF, Zhang L, Huang CF, Ma SR, Wang YF, Wang WM, Zhao ZL, Liu B, Zhao YF, Zhang WF et al: CD163 tumorassociated macrophages correlated with poor prognosis and cancer stem cells in oral squamous cell carcinoma. Biomed Res Int 2014: 838632, 2014. doi.org/10.1155/2014/838632.

41. De Palma M and Lewis CE: Macrophage regulation of tumor responses to anticancer therapies. Cancer Cell 23: 277-286, 2013. 